\title{
Inhaled NO as a Viable Antiadhesive Therapy for Ischemia/Reperfusion Injury of Distal Microvascular Beds
}

\author{
Alison Fox-Robichaud, ${ }^{\star}$ Derrice Payne, ${ }^{\star}$ Shabih U. Hasan, ${ }^{\S}$ Lena Ostrovsky, ${ }^{\star}$ Todd Fairhead, ${ }^{*}$ Paul Reinhardt, ${ }^{\star}$ \\ and Paul Kubes* \\ *Immunology Research Group, ${ }^{\ddagger}$ Division of Critical Care, and the ${ }^{\S}$ Respiratory Research Group, University of Calgary, Calgary, Alberta, \\ T2N 4N1, Canada
}

\begin{abstract}
Inhaled nitric oxide (NO) is being used more and more in intensive care units as a modality to improve the outcome of patients with pulmonary complications. Our objective was to demonstrate that inhaled NO could impact upon a distally inflamed microvasculature-improving perfusion, leukocyte adhesive interactions, and endothelial dysfunction. Using intravital microscopy to visualize ischemia/reperfusion of postcapillary venules, we were able to demonstrate that the reduction in perfusion, the dramatic increase in leukocyte rolling, adhesion, and emigration, and the endothelial dysfunction could all be significantly abrogated with $80 \mathrm{ppm}$, but not $20 \mathrm{ppm}$ inhaled NO. Perfusing whole blood directly over an inert P-selectin and CD18 ligand substratum incorporated in a flow chamber recruited the same number of rolling and adhering leukocytes from NO-ventilated and non-NO-ventilated animals, suggesting that inhaled NO was not directly affecting leukocytes. To demonstrate that inhaled NO was actually reaching the peripheral microvasculature in vivo, we applied a NO synthase inhibitor locally to the feline mesentery and demonstrated that the vasoconstriction, as well as leukocyte recruitment, were essentially abolished by inhaled NO, suggesting that a NOdepleted peripheral microvasculature could be replenished with inhaled NO in vivo. Finally, inhaled NO at the same concentration that was effective in ischemia/reperfusion did not affect vascular alterations, leukocyte recruitment, and endothelial dysfunction associated with endotoxemia in the feline mesentery. In conclusion, our data for the first time demonstrate a role for inhaled NO as a therapeutic delivery system to the peripheral microvasculature, showing tremendous efficacy as an antiadhesive, antivasoconstrictive, and antipermeabilizing molecule in NO-depleted tissues, but not normal microvessels or vessels that have an abundance of NO (LPS-treated). The notion that blood borne molecules have NO carrying capacity is conceptually consistent with
\end{abstract}

Address correspondence to Paul Kubes, Immunology Research Group, University of Calgary Medical Center, Calgary, Alberta T2N 4N1, Canada. Phone: 403-220-8558. FAX: 403-283-1267. E-mail: pkubes@acs.ucalgary.ca

Received for publication 5 January 1997 and accepted in revised form 1 April 1998.

J. Clin. Invest.

(C) The American Society for Clinical Investigation, Inc. 0021-9738/98/06/2497/09 \$2.00

Volume 101, Number 11, June 1998, 2497-2505

http://www.jci.org our observations. (J. Clin. Invest. 1998. 101:2497-2505.) Key words: endothelium • neutrophils • endotoxin • selectins • integrins

\section{Introduction}

In 1991, we proposed that endogenous, constitutively produced nitric oxide $(\mathrm{NO})^{1}$ was a homeostatic regulator of leukocyte adhesion (1). This contention was based on the fact that inhibition of endogenous NO synthesis caused leukocyte adhesion in the feline mesenteric microvasculature. This observation has been reproduced in numerous species including rats, mice, rabbits, and humans (2-5), and in various organs including lungs, liver, heart, and skeletal muscle $(2-4,6)$, suggesting this is a consistent observation across species and organ systems. Over the last 6 yr the work has been extended to suggest that NO may inhibit each of the multistep cascade of events that are involved in leukocyte recruitment including $(a)$ rapid P-selectin-dependent leukocyte rolling (a prerequisite for adhesion), (b) CD18-dependent leukocyte adhesion, and (c) ICAM-1 and VCAM-1 upregulation (1, 7-9). These data have sparked much attention, inasmuch as reductions in NO production have been reported in a number of inflammatory conditions wherein leukocyte infiltration is a key component of the pathophysiology. For example, an impairment in constitutive NO production from endothelium has been documented after ischemia/reperfusion in numerous tissues and is closely correlated with leukocyte recruitment and the ensuing vascular dysfunction $(3,10,11)$. In sepsis, despite the overproduction of NO from the inducible form of the NO synthase (iNOS; resulting in hypotension), constitutive NO production from endothelial cells has also been shown to be impaired $(12,13)$. Clearly, delivering NO to the leukocyte-endothelial cell interface of NO-deprived tissues would have tremendous therapeutic benefit.

In the last $6 \mathrm{yr}$, a significant development in the respiratory field has been the use of inhaled NO, initially in infants with pulmonary hypertension, but more recently with adults who have adult respiratory distress syndrome and pulmonary hypertension $(14,15)$. Therapeutically, the main target of inhaled $\mathrm{NO}$ is as a local vasodilator in the pulmonary vasculature. To date, there is no documentation of apparent effects on peripheral vasculatures, presumably because NO reacts very quickly with heme groups and thereby is rapidly cleared from blood. However, a systematic assessment of inhaled NO on peripheral vasculatures by actually visualizing these microvessels has never been performed. Moreover, the effect of inhaled NO on peripheral vascular beds with depleted or lowered levels of

1. Abbreviations used in this paper: iNOS, inducible NO synthase; $\mathrm{NO}$, nitric oxide; PAF, platelet-activating factor; SMA, superior mesenteric artery; $V_{\mathrm{RBC}}$, red blood cell velocity. 
NO has also never been assessed. These issues must be addressed in light of recent work by Loscalzo and colleagues and Stamler and colleagues who have suggested that NO also undergoes nitrosylation with protein-bound thiol groups under physiologic conditions producing stable S-nitrosoproteins (16-18). These investigators proposed that protein thiols can serve as an NO adduct preserving bioactivity and increasing the half-life of NO in biological systems. If this is the case, then continuous delivery of NO to blood in the form of inhaled NO could conceivably produce these and other NO adducts and thereby steadily deliver small amounts of NO to peripheral tissues. Data regarding this issue could provide insight into the use of inhaled NO and conceivably alter management of patients with reperfusion injury associated with the heart, brain, and other tissues.

Therefore, the objective of this study was to visualize and systematically assess for the first time the effects of inhaled NO on vasoactivity, leukocyte-endothelial cell interactions, and endothelial dysfunction in the peripheral microvasculature of NO depleted vessels, as well as postischemic and septic vessels. In addition, we assessed whether inhaled NO was directly affecting the ability of leukocytes to tether, roll, and adhere to substratum not exposed to inhaled NO. Our results for the first time reveal profound effects of inhaled NO upon a distal microvascular bed exposed to a NO inhibitor or ischemia/reperfusion, specifically impacting upon the key features of the inflammatory response, leukocyte-endothelial cell interactions, endothelial dysfunction, and microvascular blood flow. Additionally, we report no effect of inhaled NO on leukocyteendothelial cell interactions and vascular dysfunction in LPStreated vessels, suggesting efficacy of inhaled NO in some but not all inflammatory settings.

\section{Methods}

Surgery for intravital microscopy. The experimental preparation used in this study is the same as described previously (19). Animal protocols were approved by the University of Calgary Animal Care Committee and met the Canadian Guidelines for Animal Research. Briefly, cats (1.2-2.4 kg) were fasted for $24 \mathrm{~h}$ and initially anesthetized with ketamine hydrochloride ( $75 \mathrm{mg}$, intramuscularly). The jugular vein was cannulated and anesthesia was maintained by the administration of pentobarbital sodium. A tracheotomy was performed to support breathing by artificial ventilation. $\mathrm{NO}$ at 0,20 , or $80 \mathrm{ppm}$ was delivered from a certified grade $\mathrm{NO} /$ Balance $\mathrm{N}_{2}$ gas cylinder, to the inhalation line of a Harvard ventilator via a high accuracy flowmeter (Matheson Gas Products Canada, Edmonton, Alberta, Canada), and $\mathrm{NO}$ and $\mathrm{NO}_{2}$ were rapidly and accurately measured with a Pulmonox $11 \mathrm{NO}$ and $\mathrm{NO}_{2}$ electrochemical analyzer (Pulmonox Research and Development Corp., Tofield, Alberta, Canada). Throughout the experiments, exhaled $\mathrm{NO}_{2}$ was $<5 \mathrm{ppm}$ and was not different among groups. This NO delivery setup was similar to the one used to deliver NO to newborn infants with respiratory distress in the neonatal intensive care unit of the Foothills Medical Centre (University of Calgary, Calgary, Alberta, Canada), except in our system the cats were not provided with supplemental oxygen but rather ventilated on room air. Systemic arterial pressure was monitored by a pressure transducer (Statham P23A; Gould Inc., Oxnard, CA) connected to a catheter in the left carotid artery. A midline abdominal incision was made and a segment of small intestine was isolated from the ligament of Treitz to the ileocecal valve. The remainder of the small and large intestine was extirpated. Body temperature was maintained at $37^{\circ} \mathrm{C}$ using an infrared heat lamp. All exposed tissues were moistened with saline-soaked gauze to prevent evaporation. Heparin sodium $(10,000 \mathrm{U}$,
Elkins-Sinn, Inc., Cherry Hill, NJ) was administered, then an arterial circuit was established between the superior mesenteric artery (SMA) and left femoral artery. SMA blood flow was continuously monitored using an electromagnetic flowmeter (Carolina Medical Electronics, Inc., King, NC). Blood pressures were continuously recorded with a physiological recorder (Grass Instruments Co., Quincy, MA).

Cats were placed in a supine position on an adjustable Plexiglas microscope stage and a segment of mid-jejunum was exteriorized through the abdominal incision. The mesentery was prepared for in vivo microscopic observation, as previously described (19). The mesentery was draped over an optically clear viewing pedestal that allowed for transillumination of a $3-\mathrm{cm}$ segment of tissue. The temperature of the pedestal was maintained at $37^{\circ} \mathrm{C}$ with a constant temperature circulator (model 80; Fisher Scientific Co., Pittsburgh, PA). The exposed bowel was draped with saline-soaked gauze, whereas the remainder of the mesentery was covered with Saran Wrap $^{\circledR}$ (Dow Corning Corp., Midland, MI). The exposed mesentery was suffused with warmed bicarbonate-buffered saline $(\mathrm{pH} 7.4)$ that was bubbled with a mixture of $5 \% \mathrm{CO}_{2}$ and $95 \% \mathrm{~N}_{2}$. The mesenteric preparation was observed through an intravital microscope (Optiphot-2; Nikon Inc., Mississauga, Canada) with a $\times 25$ objective lens (Wetzlar L25/0.35; E. Leitz Inc., Munich, Germany) and a $\times 10$ eyepiece. The image of the microcirculatory bed $(\times 1,400)$ was recorded using a video camera (Digital 5100; Panasonic, Osaka, Japan) and a video recorder (NV8950; Panasonic).

Single unbranched mesenteric venules (25-40- $\mu \mathrm{m}$ diameter, 250$\mu \mathrm{m}$ length) were selected for each study. Venular diameter was measured on- or offline using a video caliper (Microcirculation Research Institute, Texas A\&M University, College Station, TX). The number of rolling and adherent leukocytes was determined offline during playback analysis. Rolling leukocytes were defined as white blood cells that moved at a velocity less than that of erythrocytes in a given vessel. The number of rolling leukocytes (flux) was counted using frame by frame analysis. To obtain a complete leukocyte rolling velocity profile, the rolling velocity of all leukocytes entering the vessel was measured. A leukocyte was defined as adherent to venular endothelium if it remained stationary for $>30 \mathrm{~s}$. Adherent cells were measured at 10-min intervals as described in the experimental proto$\mathrm{col}$, and expressed as the number per 100- $\mu \mathrm{m}$ length of venule. Red blood cell velocity $\left(V_{\mathrm{RBC}}\right)$ was measured using an optical Doppler velocimeter (Microcirculation Research Institute) and mean red blood cell velocity $\left(V_{\text {mean }}\right)$ was determined as $V_{\mathrm{RBC}} / 1.6(20)$. Wall shear rate was calculated based on the Newtonian definition: shear rate $=$ $\left(V_{\text {mean }} / D v\right) \times\left(8 s^{-1}\right)$, where $\mathrm{Dv}$ is the venular diameter.

Microvascular permeability. The degree of microvascular dysfunction was assessed as vascular albumin leakage in cat mesenteric venules. Briefly, $25 \mathrm{mg} / \mathrm{kg}$ FITC-labeled bovine albumin (Sigma Chemical Co., St. Louis, MO) was administered intravenously to animals 15 min before the start of the experimental procedure. Fluorescence intensity (excitation wavelength, $420-490 \mathrm{~nm}$; emission wavelength, $520 \mathrm{~nm}$ ) was detected using a silicon-intensified fluorescent camera (model C-2400-08; Hamamatsu Photonics, Hamamatsu, Japan) and images were recorded for playback analysis using a video cassette recorder. The fluorescent intensity of FITC-albumin within a defined area $(10 \mu \mathrm{m} \times 50 \mu \mathrm{m})$ of the venule under study and in the adjacent perivascular interstitium ( $20 \mu \mathrm{m}$ from venule) was measured during the control period, at $60 \mathrm{~min}$ of ischemia, and at 10 and $60 \mathrm{~min}$ of reperfusion. This was accomplished using a video capture board (Visionplus AT-OFG; Imaging Technology Inc., Bedford, MA) and a computer-assisted digital imaging processor (Optimas; Bioscan Inc., Edmonds, WA). The index of vascular albumin leakage (permeability index) was determined from the ratio: (interstitial intensity background $) /($ venular intensity - background $)$, as previously reported $(21,22)$.

Experimental protocol. Baseline measurements of blood pressure, SMA blood flow, $\mathrm{V}_{\mathrm{RBC}}$, and vessel diameter were obtained. Experiments were carried out at either 0,20 , or $80 \mathrm{ppm} \mathrm{NO}$ in addition to 
the room air ventilation. In this first series of experiments, the preparation was videotaped for $10 \mathrm{~min}$, and then SMA blood flow was mechanically reduced (Gaskell clamp) to $20 \%$ of control for $1 \mathrm{~h}$. The last $10 \mathrm{~min}$ of the ischemic period were videotaped and then the clamp was removed to restore intestinal blood flow. Video recordings were made at 10,60, and 120 minutes after reperfusion.

To further evaluate the role of inhaled $\mathrm{NO}$ as a molecule that could affect the distal microvasculature, in some experiments animals received 0 or $80 \mathrm{ppm}$ NO. Then the mesenteric microvasculature was locally superfused with the NO inhibitor N $\omega$-nitro-L-arginine-methyl ester $\mathrm{HCl}$ (L-NAME; $100 \mu \mathrm{M}$ for $120 \mathrm{~min}$ ), which has been shown previously to cause vasoconstriction and increased leukocyte rolling and adhesion and emigration. Finally, to examine the role of inhaled NO on a vasculature wherein iNOS is induced and presumably excessive NO is being produced, animals again inhaled either 0 or $80 \mathrm{ppm}$ NO, but the mesenteric microvasculature was superfused with lipopolysaccharide (Escherichia coli $250 \mathrm{ng} / \mathrm{ml}$ ) for $4 \mathrm{~h}$. We have previously detected substantial iNOS induction at $2-4 \mathrm{~h}$ of this protocol (23).

Platelet isolation and monolayer preparation. We and others (24-26) previously established that perfusion of neutrophils over platelet monolayers using flow chambers (described below) caused P-selectin-dependent leukocyte rolling and platelet-activating factor (PAF)dependent leukocyte adhesion. This was important as it provided exactly the substratum needed to mimic the adhesive mechanisms previously reported for the leukocyte rolling and adhesion after ischemia/reperfusion $(19,27)$. Feline platelets were isolated from donors by spinning the whole blood at $150 \mathrm{~g}$ for $10 \mathrm{~min}$ at room temperature. The supernatant ( $\sim 10 \mathrm{ml}$ from $30 \mathrm{ml}$ of whole blood) was reconstituted to $50 \mathrm{ml}$ with $2 \mathrm{ml}$ of acetate-citrate-dextrose anticoagulant and phosphate-buffered saline and spun at room temperature for $10 \mathrm{~min}$ at $1,000 \mathrm{~g}$. The supernatant was discarded and the pellet resuspended in $5 \mathrm{ml}$ of HBSS. The platelets were diluted to a final concentration of $2 \times 10^{8}$ platelets $/ \mathrm{ml}$ with HBSS, and $1 \mathrm{ml}$ was placed on coverslips that had been incubated with collagen type 1 for $1 \mathrm{~h}$ at $37^{\circ} \mathrm{C}$. The cells were allowed to settle for $1 \mathrm{~h}$ at $37^{\circ} \mathrm{C}$. The coverslips were rinsed leaving a confluent monolayer of platelets.

Protocol. To establish that neutrophils were not directly affected by inhaled NO, the circulating leukocytes that passed through lungs exposed to inhaled NO had to then be introduced to unalterable levels of feline P-selectin and PAF. Therefore, in all of these experiments, the platelet monolayers were fixed ( $1 \%$ neutral buffered formalin). This also allowed the use of monolayers from the same preparation on consecutive days of experimentation with and without inhaled NO. Fixation had no effect on leukocyte rolling and only minor effects on firm adhesion (24), allowing for storage and use of monolayers prepared on the same day from the same cat in multiple experiments. A Perspex parallel-plate flow chamber identical to the one described by Lawrence et al. (28) was used. The platelet monolayers were used as the chamber's bottom plate. The flow chamber was placed onto the stage of an inverted microscope (Carl Zeiss, Don Mills, Ontario, Canada) and leukocyte interactions were visualized at $100 \times$ using phase-contrast microscopy. The stage area was enclosed in a warm air cabinet and maintained at $37^{\circ} \mathrm{C}$. Whole blood (diluted 1:10 with HBSS) from cats was drawn via a syringe pump (Harvard Apparatus, St. Laurent, Quebec, Canada) through the flow chamber at $37^{\circ} \mathrm{C}$ and 4.0 dynes $/ \mathrm{cm}^{2}$.

Unlike previous flow chamber experiments where leukocytes were isolated, the delay during isolation was not suitable to determine whether inhaled NO was affecting leukocytes directly, so whole blood was perfused immediately over the platelet monolayers for 5 min, and then HBSS was chased for an additional 5 min to flush out erythrocytes and noninteracting leukocytes. Although leukocyte interactions could not be adequately visualized during blood perfusion, once HBSS was introduced, the leukocytes interacting with the coverslip could be counted easily. Experiments were filmed with a CCD video camera (Hitachi Denshi, San Jose, CA) and recorded on a video cassette recorder for playback analysis. The number of interact- ing leukocytes was counted in at least four random fields per coverslip, and expressed as the number of interacting cells $/ \mathrm{mm}^{2}$. To identify the type of leukocytes recruited, at the end of the experiment, the coverslips were gently removed, air-dried, and stained in Diff-Quik ${ }^{\circledR}$ (Baxter International Inc., Miami, FL) according to the manufacturer's instructions, mounted on a slide, and examined microscopically. Blood $(2 \mathrm{ml})$ was taken from anesthetized cats that only had a catheter placed into the carotid to monitor blood pressure. The animals were then ventilated with either 0 or $80 \mathrm{ppm}$ NO for $6 \mathrm{~h}$ (approximate length of ischemia/reperfusion experiments). Blood $(2 \mathrm{ml})$ was taken at the end of the experiments, and again instantly perfused over a platelet monolayer as described above.

Statistics. The data were analyzed using standard statistical analysis, i.e., ANOVA and Student's $t$ test with Bonferroni's correction for multiple comparisons where appropriate. All values are mean \pm SEM. Statistical significance was set at $P<0.05$.

\section{Results}

Table I summarizes the results of blood pressure, venular diameter, and $V_{\mathrm{RBC}}$ before and $120 \mathrm{~min}$ after ischemia/reperfusion in animals inhaling 0,20 , or $80 \mathrm{ppm}$ NO. The blood pressure was not different between animals ventilated on room air alone and those ventilated with room air plus NO, consistent with the view that inhaled NO does not affect resistance in distal vascular beds under control conditions. Table I extends these observations to reveal no difference of inhaled NO on microvascular parameters including venular diameter and $V_{\mathrm{RBC}}$ between groups under baseline conditions. Table I also demonstrates that blood pressure remains within $15 \%$ of control levels throughout the time of reperfusion (only 2-h time point shown). Within the microvasculature, venular diameters did not change during ischemia or the reperfusion period in any of the groups consistent with previous observations. On the other hand, $V_{\mathrm{RBC}}$ were reduced to $50 \%$ of the preischemic values in untreated animals. When the animals were ventilated with $80 \mathrm{ppm} \mathrm{NO}$, the reperfusion-induced decrease in $V_{\mathrm{RBC}}$ was dramatically reduced.

Intestinal blood flow after reperfusion rapidly rises above control due to a hyperemic response (not shown) and by 10 min begins to drop below control values and progressively decreases thereafter (Fig. 1). Whereas the blood pressure response to reperfusion did not differ between the three $(0,20$, and $80 \mathrm{ppm}$ ) groups of animals, the intestinal blood flow was

Table I. Hemodynamic Effects of Inhaled NO before and after Ischemia

\begin{tabular}{lrcc}
\hline & & $\begin{array}{c}\text { Before } \\
\text { ischemia }\end{array}$ & $\begin{array}{c}120 \mathrm{~min} \\
\text { after reperfusion }\end{array}$ \\
\hline Mean blood pressure $(\mathrm{mmHg})$ & $0 \mathrm{ppm}$ & $107 \pm 4$ & $93 \pm 4^{*}$ \\
& $20 \mathrm{ppm}$ & $100 \pm 8$ & $102 \pm 7$ \\
& $80 \mathrm{ppm}$ & $108 \pm 7$ & $108 \pm 7$ \\
Venular diameter $(\mu \mathrm{m})$ & $0 \mathrm{ppm}$ & $38.0 \pm 1.4$ & $38.3 \pm 1.1$ \\
& $20 \mathrm{ppm}$ & $38.3 \pm 0.6$ & $39.3 \pm 0.5$ \\
$V_{\mathrm{RBC}}(\mu \mathrm{m} / \mathrm{s})$ & $80 \mathrm{ppm}$ & $38.0 \pm 1.0$ & $38.6 \pm 1.3$ \\
& $0 \mathrm{ppm}$ & $4.0 \pm 0.3$ & $1.7 \pm 0.3^{*}$ \\
& $20 \mathrm{ppm}$ & $3.9 \pm 0.2$ & $2.5 \pm 0.6$ \\
& $80 \mathrm{ppm}$ & $4.1 \pm 0.2$ & $3.6 \pm 0.6^{* \ddagger}$
\end{tabular}

${ }^{*} P<0.05$ relative to control; ${ }^{\ddagger} P<0.05$ relative to untreated $(n=18$ total). 

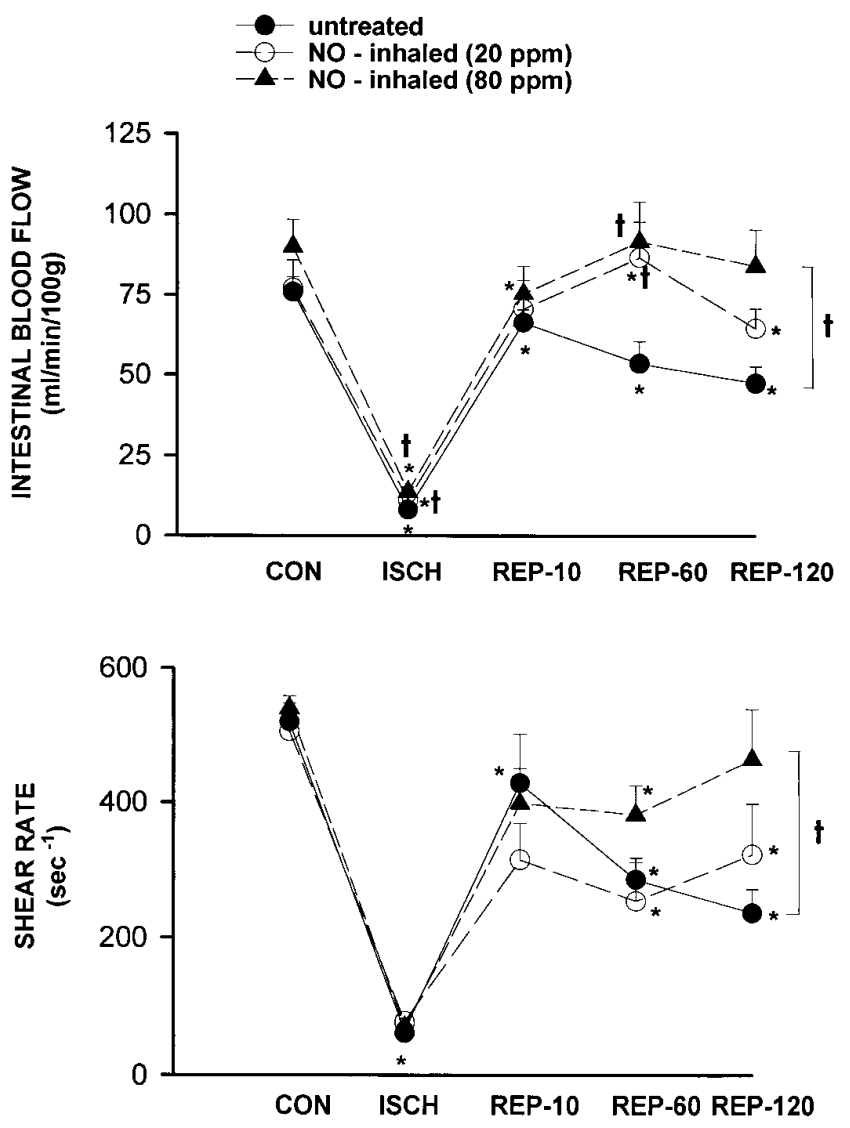

Figure 1. Intestinal blood flow (top) and shear rate (bottom) during control $(C O N)$, ischemia (ISCH) and at 10, 60, and 120 min after reperfusion $(R E P)$ in untreated cats $(n=6)$, and cats inhaling 20 $(n=4)$ or $80 \mathrm{ppm} \mathrm{NO}(n=8) .{ }^{*} P<0.05$ relative to control; ${ }^{\dagger} P<0.05$ relative to untreated group.

maintained at preischemic values for the first $60 \mathrm{~min}$ in the 20ppm group and then decreased by $120 \mathrm{~min}$. At the higher concentration of NO (80 ppm), intestinal blood flow was maintained at preischemic values throughout the experiment (top). During reperfusion the calculated shear rates were reduced to $50 \%$ of preischemic values (bottom). Again, when the animals were ventilated with $80 \mathrm{ppm} \mathrm{NO}$, the $50 \%$ reduction in reperfusion-induced decrease in shear rate was almost entirely prevented. At $20 \mathrm{ppm}$ there was still a significant decrease in this parameter.

Fig. 2 illustrates the changes in leukocyte rolling flux and leukocyte rolling velocity during ischemia/reperfusion. Leukocyte rolling flux in feline mesenteric microvasculature is consistently between 45 and 60 cells/min. This value is decreased during the ischemic episodes as many of the cells tend to remain stationary during the very low flow state (not shown). At the time of reperfusion there is a very profound increase in leukocyte rolling flux to 200 to 250 cells/min and this value is maintained throughout the $2 \mathrm{~h}$ of reperfusion. Neither 20 or 80 ppm NO had an effect on baseline leukocyte rolling and in every case there was a dramatic increase in leukocyte rolling at $10 \mathrm{~min}$ of reperfusion. However, by 60 to $120 \mathrm{~min}$ there was a significant $(30-40 \%)$ reduction in rolling flux. In addition to the increased rolling response after ischemia, there is also a very dramatic decrease in rolling velocity. This is extremely critical as the lower rolling velocity permits cells to respond to very low levels of stimulus, an event that does not occur in faster rolling leukocytes (29). It is noteworthy that neither level of NO inhalation had any effect on leukocyte rolling velocity after reperfusion, suggesting that not all aspects of leukocyte behavior were altered with inhaled NO during reperfusion.

The most striking effects of inhaled NO are illustrated in Fig. 3, which summarizes the adhesion and emigration data. In animals breathing room air, leukocyte adhesion increases from $<1$ cell/100 $\mu \mathrm{m}$ venule length to $\sim 15-20$ cells $/ 100 \mu \mathrm{m}$ venule length adhering during the first $2 \mathrm{~h}$ of reperfusion. The adhering cells progressively migrate out of the vasculature (Fig. 3, bottom), such that by $120 \mathrm{~min}$ there is a $>40$-fold increase in the number of emigrated cells. Neither adhesion nor emigration is affected by inhaled NO at the lower dose. Within the first $10 \mathrm{~min}$ of reperfusion, the higher dose of NO had no effect on adhesion or emigration, although there is little emigration at this time point. However, over the next $120 \mathrm{~min}$, adhesion was dramatically reduced and the emigration was entirely arrested with $80 \mathrm{ppm}$ NO. Associated with the increase in leuko-
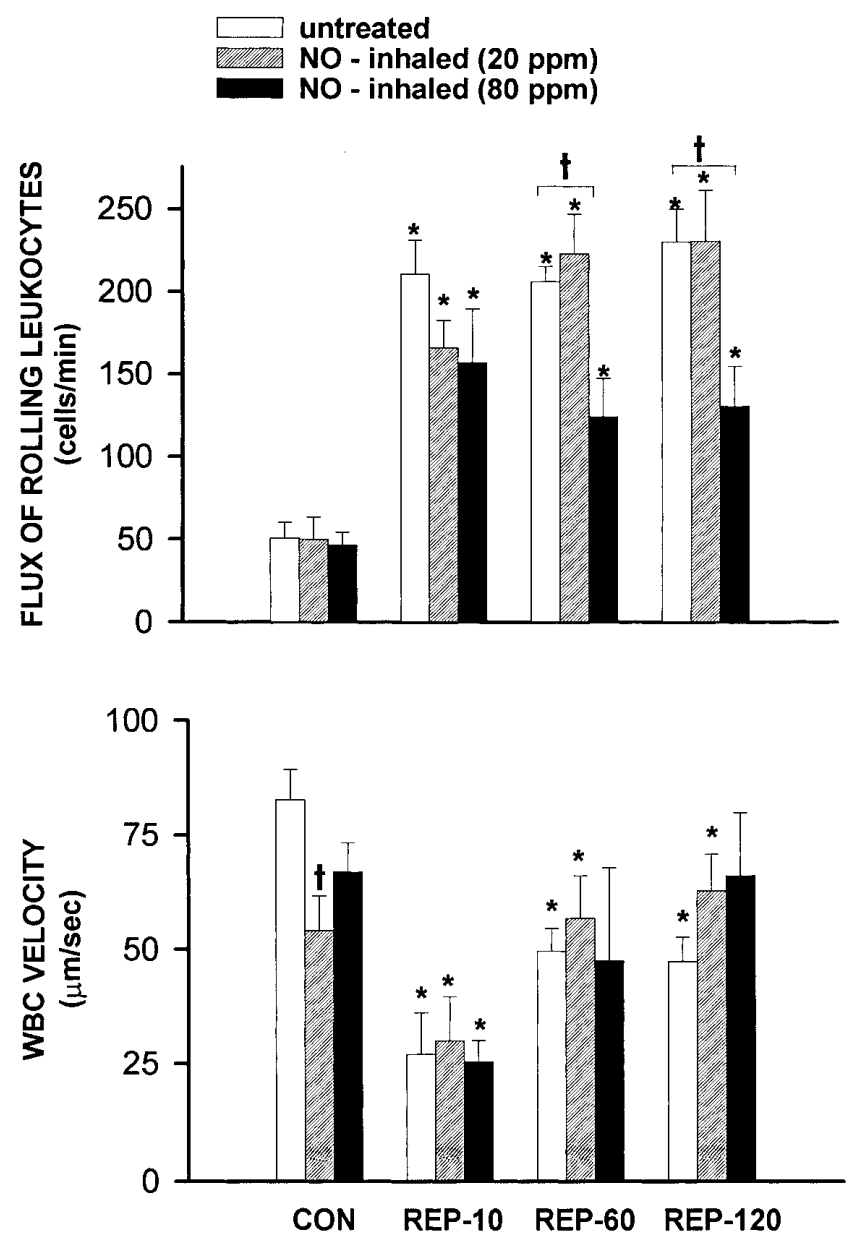

Figure 2. Leukocyte rolling flux (top) and white blood cell (WBC) velocity (bottom) during control period $(C O N)$ and 10, 60, and 120 min of reperfusion $(R E P)$ in animals untreated $(n=6)$, or inhaling 20 $(n=4)$ or $80 \mathrm{ppm} \mathrm{NO}(n=8) .{ }^{*} P<0.05$ relative to control; ${ }^{\dagger} P<0.05$ relative to untreated group. 

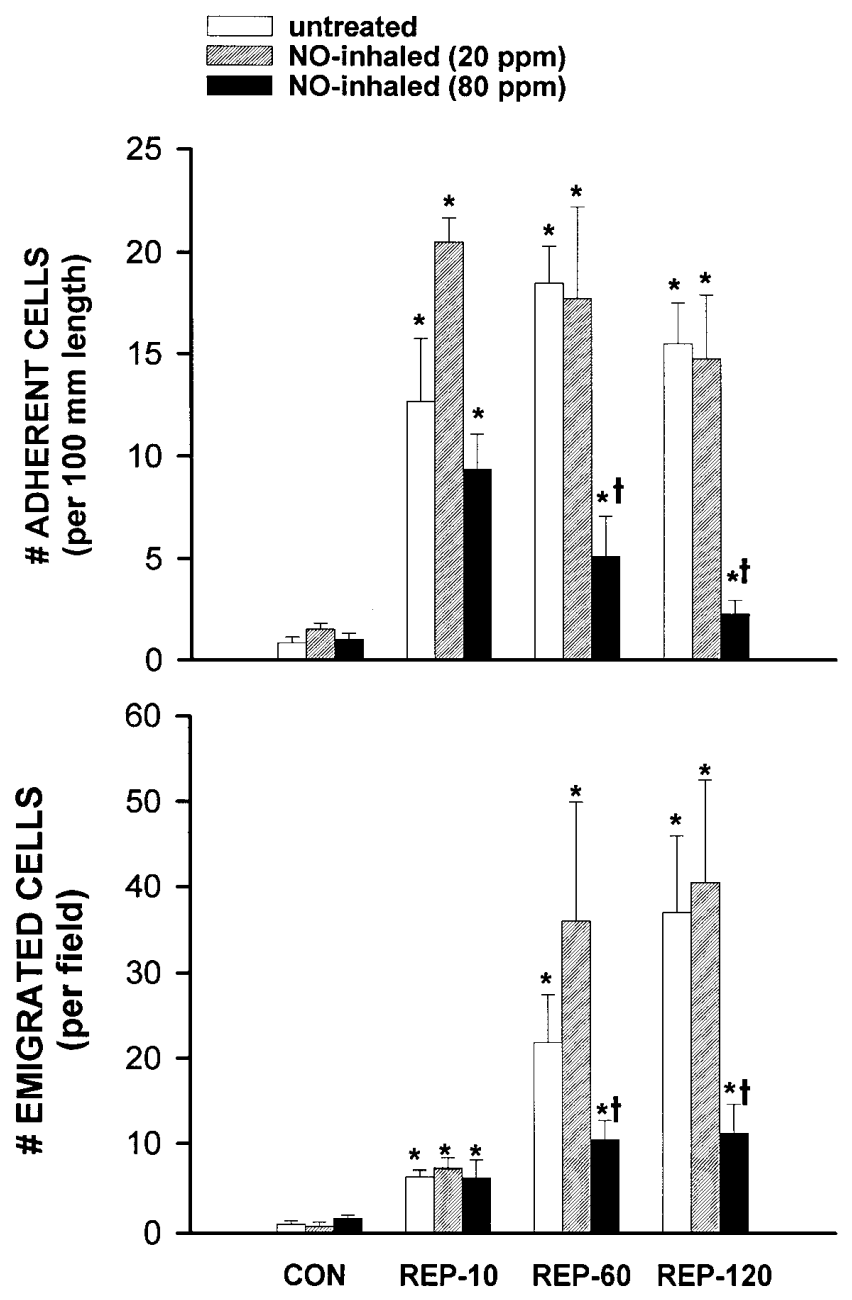

Figure 3. Adhesion (top) and emigration (bottom) of leukocytes during control period $(C O N)$ and 10,60 , and $120 \mathrm{~min}$ after reperfusion $(R E P)$ in untreated cats $(n=6)$, or cats inhaling $20(n=4)$ or $80 \mathrm{ppm}$ NO $(n=8) .{ }^{*} P<0.05$ relative to control; ${ }^{\dagger} P<0.05$ relative to untreated group.

cyte adhesion and emigration in reperfused tissue was a very dramatic increase in FITC-albumin leakage out of the vasculature (Fig. 4). Inhaled NO (80 ppm but not $20 \mathrm{ppm}$ ) significantly reduced the endothelial dysfunction in response to ischemia/reperfusion by close to $70 \%$ at $60 \mathrm{~min}$ of reperfusion and by $50 \%$ at $120 \mathrm{~min}$ after ischemia. For the remaining experiments, only the higher concentration of inhaled NO was used.

Inhaled NO does not directly affect activation/adhesion of leukocytes. To establish whether inhaled NO was affecting leukocytes as they passed through the lung, as opposed to reaching peripheral tissue, we allowed animals to breath NO for $6 \mathrm{~h}$ (length of ischemia/reperfusion experiment including surgery) and then examined leukocyte rolling and adhesion on a feline substratum (immobilized platelet monolayers) that most closely mimicked reperfusion-induced, P-selectin-dependent rolling and PAF/CD18-dependent leukocyte adhesion. Blood taken from cats and perfused immediately over platelet monolayers resulted in leukocyte rolling and adhesion that could easily be visualized once the whole blood was chased with HBSS (Fig. 5). Blood from animals that were breathing 80 ppm NO revealed identical rolling and adhesive properties, suggesting that inhaled NO did not affect the leukocytes' ability to interact with P-selectin to roll or CD18-ligand to adhere.

Inhaled NO can replenish a NO-depleted tissue. To demonstrate that NO was reaching the peripheral vasculature, we inhibited NO locally within the mesentery by superfusing L-NAME. This causes arteriolar vasoconstriction and increases in neutrophil rolling and adhesion and emigration. Fig. 6 demonstrates that L-NAME caused an $\sim 9-\mu \mathrm{m}$ decrease in the diameter of these small precapillary vessels after $2 \mathrm{~h}$ of superfusion. Inhaled NO at $80 \mathrm{ppm}$ completely prevented the ability of L-NAME to induce vasoconstriction in the mesenteric microvasculature. Leukocyte-endothelial cell interactions are dramatically increased in the presence of L-NAME: leukocyte rolling increases from 50 to 150 cells/min, leukocyte adhesion increases from 1 cell $/ 100 \mu \mathrm{m}$ length venule to 15 cells $/ 100 \mu \mathrm{m}$ length venule and leukocyte emigration increases $\sim 15$-fold (Fig. 7). At 80 ppm inhaled NO, these responses were not apparent in any vessel examined. The preparations were identical to untreated microvessels.

Inhaled NO does not affect a NO replete tissue. In a previous study, we have demonstrated that superfusion of the cat mesentery with LPS induces iNOS as well as leukocyte recruitment and vascular dysfunction (23). Fig. 8 highlights data suggesting that inhaled NO does not affect microvascular hemodynamic factors, leukocyte accumulation, or endothelial cell dysfunction associated with inhaled NO. The top bar graph demonstrates that LPS decreases venular shear rates over $4 \mathrm{~h}$. The middle demonstrates a dramatic increase in leukocyte recruitment (emigration), and the bottom outlines the increase in microvascular dysfunction. None of these parameters were

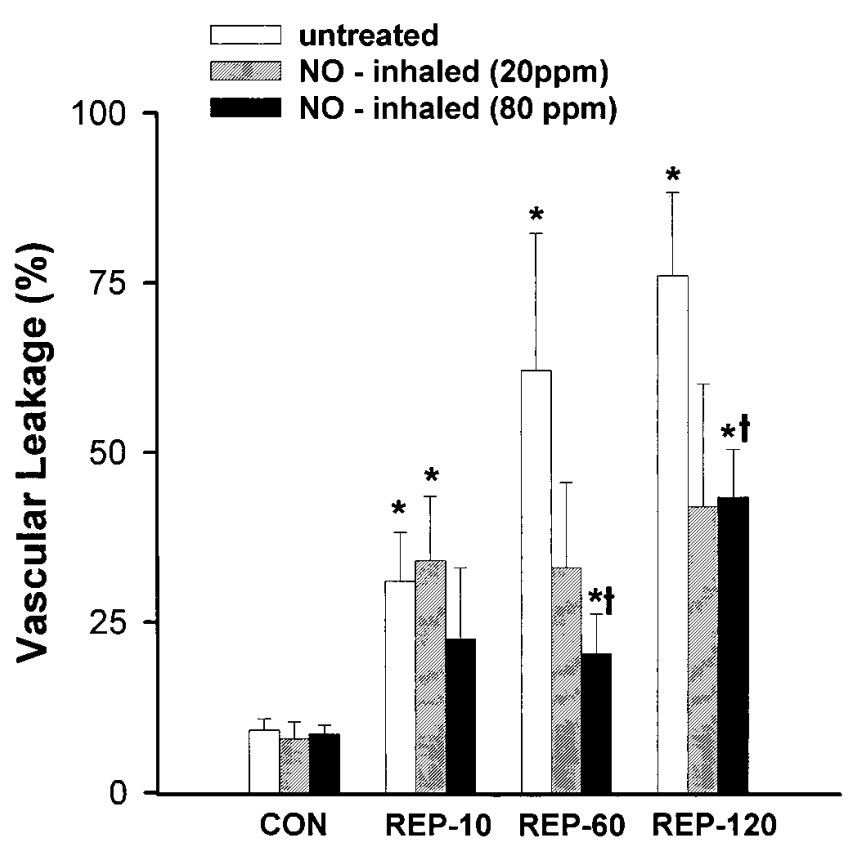

Figure 4. Vascular leakage as determined by extravasation of FITCalbumin during control period $(C O N)$ and 10, 60, and 120 min after reperfusion $(R E P)$ in untreated cats, or cats inhaling 20 or $80 \mathrm{ppm}$ NO. $* P<0.05$ relative to control; ${ }^{\dagger} P<0.05$ relative to untreated group. 

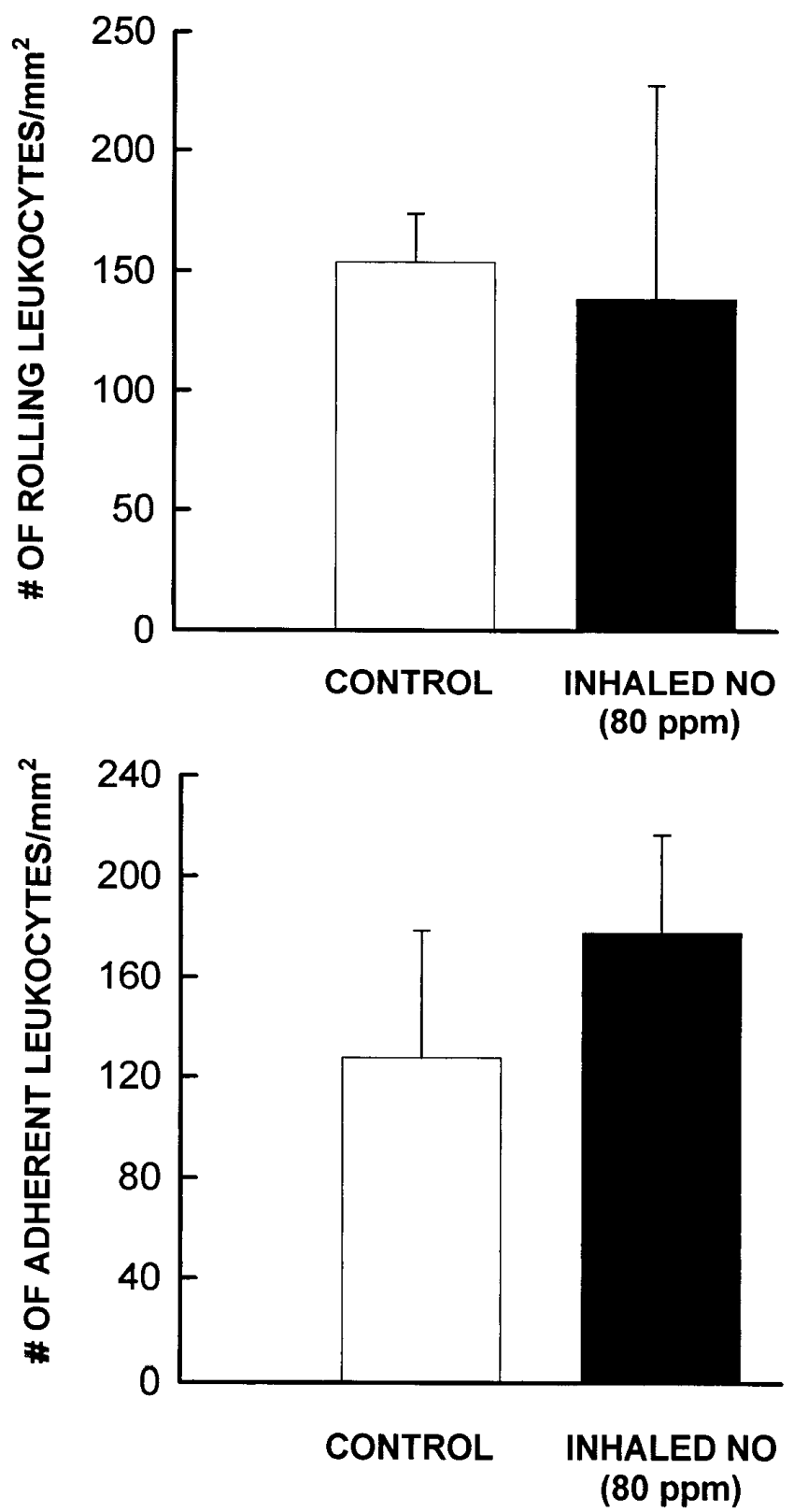

Figure 5. Leukocyte rolling (top) and adhesion (bottom) of whole blood leukocytes over a fixed platelet monolayer from cats ventilated with room air only $(C O N T R O L)$ or with $80 \mathrm{ppm}$ inhaled NO.

significantly altered by inhaled NO. In addition, inhaled NO had little effect on the rolling flux and leukocyte rolling velocity (data not shown). Inhaled $\mathrm{NO}$ at $80 \mathrm{ppm}$ caused a small reduction in leukocyte adhesion (data not shown) but, as already noted, this did not translate into a physiologically relevant reduction in extravascular leukocyte accumulation or microvascular dysfunction.

\section{Discussion}

Inhaled NO is becoming an increasingly popular therapeutic maneuver in pediatric and adult respiratory failure. In this study, we demonstrate for the first time that inhaled NO ex- tends far beyond the effects of the pulmonary vasculature to affect a peripheral microvascular bed depleted of NO. NO inhalation supplements a distal microvasculature with NO so that poor perfusion, the multistep leukocyte recruitment paradigm, and microvascular dysfunction can all be significantly abrogated. NO inhalation had absolutely no effect on the normal unperturbed microvasculature, suggesting perhaps that peripheral effects of inhaled $\mathrm{NO}$ will only be apparent in a NO-deficient vascular bed. If indeed this is the case, then NO delivery via this route should be effective in disease conditions, wherein a reduction in $\mathrm{NO}$ is a contributing factor to the pathology. Indeed, in a situation like ischemia/reperfusion, where numerous laboratories have shown a decrease in NO production as early as $2.5 \mathrm{~min}$ after reperfusion and by $>90 \%$ within 60 min of reperfusion $(3,10,11)$, inhaled NO was a very effective inhibitor of poor perfusion, of leukocyte recruitment, and microvascular dysfunction. In contrast, when the mesentery was superfused with LPS, a model wherein iNOS is induced and an abundance of NO is produced (23), inhaled NO at a level that dramatically reduced the sequelae of reperfusion injury, had absolutely no effect on LPS-induced microvascular alterations. Clearly, our data suggest that inhaled NO may benefit reperfusion type injuries without necessarily impacting upon sepsis-associated disease.

Because inhaled NO does not affect systemic blood pressure, the prevailing view to date has been that inhaled NO reaches the pulmonary microvasculature and then reacts very quickly with heme groups, thereby allowing for rapid clearance of NO from blood (30). It has, therefore, been inferred that inhaled NO would have no impact on nonpulmonary vascular beds. Indeed, neither cerebral blood flow (31) nor myocardial blood flow (32) was affected by NO inhalation (20 and $80 \mathrm{ppm}$, respectively) under normal conditions and, in the latter study, inhaled NO did not improve coronary vasoconstric-

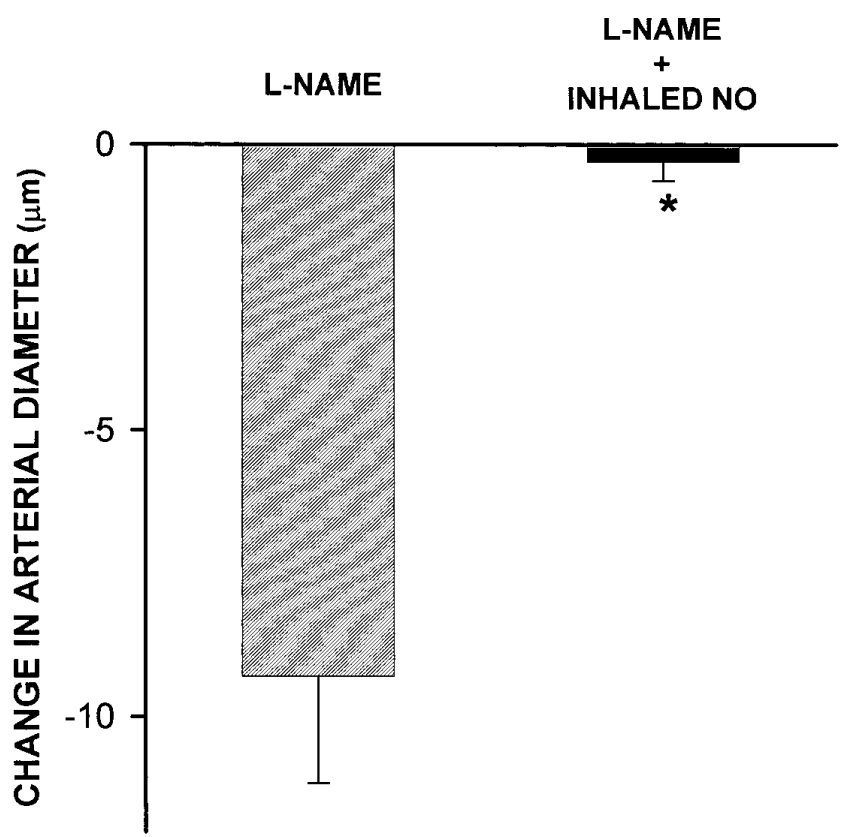

Figure 6. Change in arterial diameter at $2 \mathrm{~h}$ from baseline control period in cat mesentery superfused with L-NAME alone or L-NAME + 80 ppm inhaled NO. $* P<0.05$ relative to untreated group. 


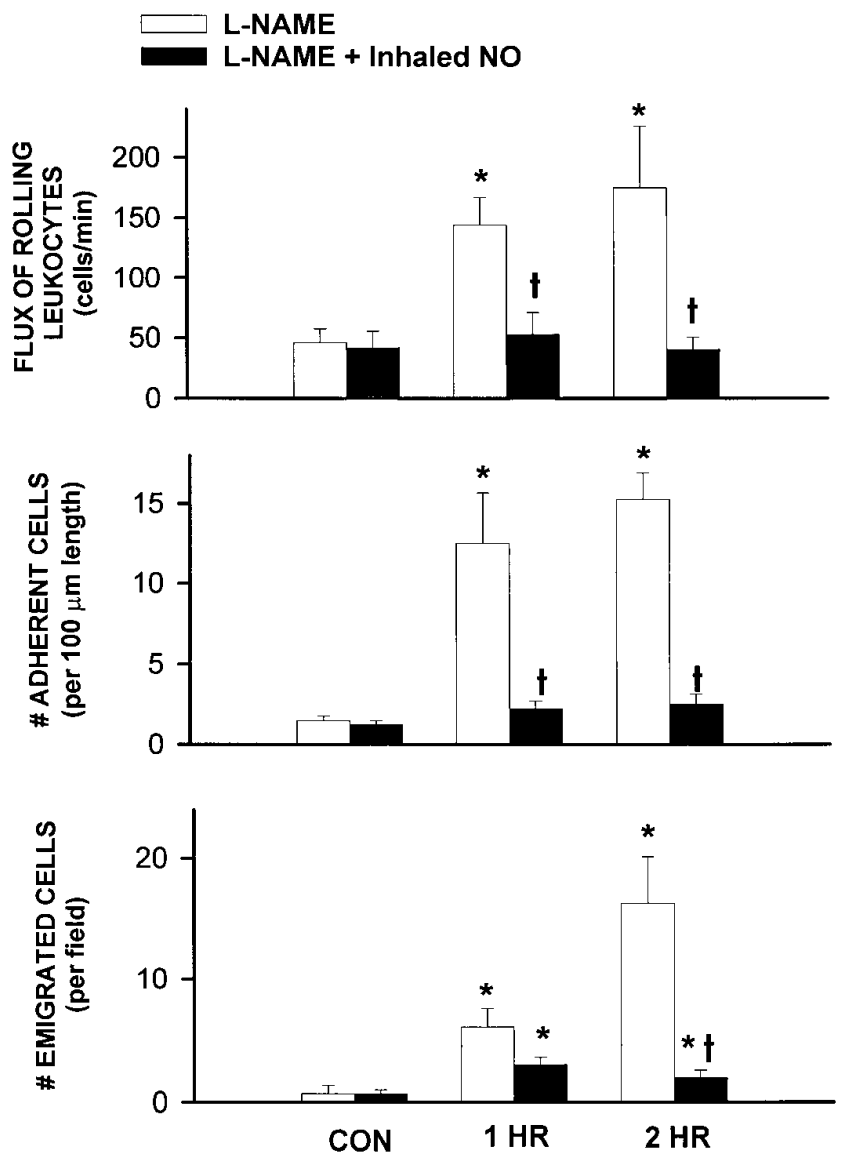

Figure 7. Leukocyte rolling flux (top), adhesion (middle), and emigration (bottom) during control period $(C O N)$ and 1 and $2 \mathrm{~h}$ after L-NAME superfusion of cat mesentery with or without $80 \mathrm{ppm}$ of inhaled NO. ${ }^{*} P<0.05$ relative to control; ${ }^{\dagger} P<0.05$ relative to untreated group.

tion in response to a thromboxane $\mathrm{A}_{2}$ agonist. Our data show that inhaled NO had absolutely no effect upon the intact peripheral microvasculature or a microvasculature that had ample NO. On the other hand, in a reperfused tissue wherein endothelial NO can be reduced by as much as $90 \%$, for example in the heart or mesentery $(3,10,11)$, inhaled $\mathrm{NO}$ provided at least as much protection after ischemia/reperfusion of a distal vascular bed, as does bolus injection or superfusion of tissues with NO donors, however, without the associated hypotension $(33,34)$. In fact, NO inhalation in a very stable and lasting manner returned blood flow to control levels and prevented all of the leukocyte emigration in the most effective way we have observed to date. Finally, microvascular permeability was reduced by $>50 \%$, which suggests that inhaled NO was actually reducing a key endpoint of reperfusion injury. Although there remained a NO-insensitive increase in microvascular permeability, it is conceivable that higher amounts of inhaled NO may have further reduced this parameter. Regardless, a 50$70 \%$ reduction in interstitial swelling in tissues such as the brain or heart with $80 \mathrm{ppm}$ inhaled NO could make a very marked difference in the outcome of stroke and myocardial infarct, where vascular dysfunction is a very real concern.

A likely mechanism of action of inhaled NO may be on transiting leukocytes at the level of the pulmonary microvasculature. This contention is based on work by a number of laboratories reporting a subtle but prolonged nonadhesive, inhibitory effect of inhaled NO on neutrophils; $5 \mathrm{~h}$ after NO inhalation in swine caused neutrophil superoxide production to be reduced (35), an effect also reported in neonates that inhaled NO for 24-72 h (36). These effects were seen after prolonged incubation times, suggesting that $\mathrm{NO}$ was having a protracted inhibitory effect upon leukocytes. However, our results clearly demonstrate that in terms of adhesive mechanisms, inhaled NO did not impair the ability of leukocytes to tether to P-selectin and firmly adhere via PAF-induced CD18 activation and adhesion even when the cells were exposed to substratum within 1-2 min of being removed from the cat. In our study, our novel whole blood perfusion in the flow chamber was designed to circumvent isolation of leukocytes, thereby exposing neutrophils with all of the blood components to the adhesive substratum. Although it could be argued that even the very short time between withdrawal of blood and perfusion into the in vitro flow chamber was sufficient to lose the NO effects upon leukocytes, much longer protocols have been used to show that inhaled NO affects platelet aggregation in some instances for as long as $5 \mathrm{~h}$ after NO inhalation (16). It is our contention that the $\mathrm{NO}$ effects were directly on the endothe-

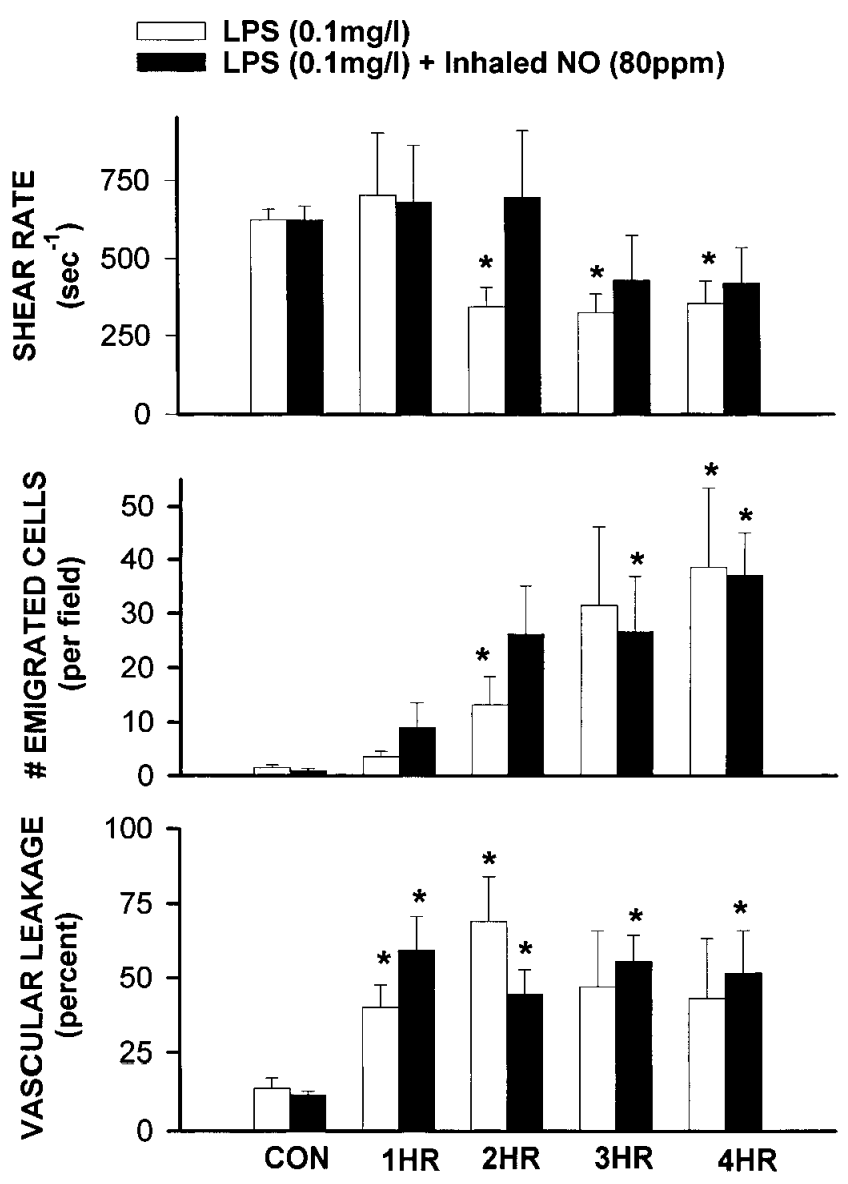

Figure 8. Shear rate (top), emigration (middle), and vascular leakage (bottom) during control period (CON) and 1,2,3, and $4 \mathrm{~h}$ after LPS superfusion with or without $80 \mathrm{ppm}$ inhaled NO. $* P<0.05$ relative to control. 
lium, a view consistent with the improved blood flow with NO inhalation in ischemia/reperfusion that is due to endothelial dysfunction, not recruited leukocytes (19).

As the reversal of blood flow with inhaled NO cannot be explained by direct effects upon leukocytes transiting through the lung, there must be a mechanism in place that stably transports NO to the periphery. NO undergoes nitrosylation with protein-bound thiol groups under physiologic conditions producing stable $S$-nitroso-proteins (17) and binding to cysteine $\beta 93$ of hemoglobin forming $S$-nitrosohemoglobin that has been proposed to release the $\mathrm{NO}$ at low oxygen tensions in the microcirculation. These nitrosothiols may represent a stabilized form of NO in biological tissues. Indeed, Keaney et al. (16) have reported that $S$-nitroso-albumin possess EDRF-like properties, including vasodilatation and inhibition of platelet aggregation. Although the responses were sevenfold less potent as a bolus than nitroprusside, these results suggest that protein thiols can serve as a NO adduct preserving bioactivity and increasing the half-life of NO in biological systems. Interestingly, a recent study has reported the production of $S$-nitrosyl-hemoglobin in sheep inhaling NO at $60 \mathrm{ppm}$ (37). Another study has reported that chronic NO inhalation could inhibit neointimal formation at a peripheral site (the carotid arteries), but this may have occurred because NO impacted upon platelets as they transited through the lungs rather than by reaching distal vasculature (38).

To directly test that inhaled NO was reaching the peripheral microvasculature, we administered a NO inhibitor locally in the mesenteric microvasculature in animals breathing 0 or $80 \mathrm{ppm}$ NO. In the former, L-NAME caused a significant vasoconstriction in precapillary arterioles and induced a substantial increase in leukocyte rolling, adhesion, and emigration. In animals ventilated with $\mathrm{NO}$, even the vasoconstrictive effects associated with inhibition of NO were entirely prevented. Although the focus of this study was not to identify the molecule that can deliver NO to the periphery, the data clearly demonstrate for the first time that inhaled NO is reaching peripheral vascular beds where it is released in NO-depleted tissues. In fact, it is tempting to propose that a NO gradient may need to be established, such that tissues lacking in NO receive a significant portion of the NO, whereas tissues replete with NO do not receive NO.

In conclusion, the data demonstrate a role for inhaled NO as a therapeutic delivery system to the peripheral microvasculature, showing tremendous efficacy in NO-depleted tissues. Secondly, our data propose this therapeutic approach as a potential means of treating reperfusion injury associated with trauma, as well as perhaps after myocardial infarct and stroke. Preliminary work from our laboratory suggests that ischemia/ reperfusion-induced poor perfusion, leukocyte recruitment, and endothelial dysfunction can also be reversed with inhaled $\mathrm{NO}$ giving further credence to its therapeutic potential. Finally, our data do not reveal a direct effect of inhaled NO upon leukocytes, or intact microvasculatures and injured microvasculatures that have increased NO production. It is noteworthy that addition of NO to our LPS model did not benefit the distal microvasculature, but also revealed no detrimental effects under these conditions. Whether this relates to ischemia/reperfusion being an oxidant-dependent model of leukocyte recruitment and therefore inhibitable by NO versus LPS, which may have less oxidant-dependent injury, remains to be established. Finally, the fact that inhaled NO greatly improves reperfusion- type injuries, but does not have detrimental or beneficial effects in sepsis, may explain why this approach has had limited success in clinical trials wherein both types of patients may be included.

\section{Acknowledgments}

The authors would like to acknowledge the technical support of Kassim Daya (Foothills Medical Center, Calgary, Alberta, Canada).

P. Kubes is a Medical Research Council Scientist and Senior Scholar of the Alberta Heritage Foundation for Medical Research. This study was supported by a grant from the Canadian Heart and Stroke Foundation.

\section{References}

1. Kubes, P., M. Suzuki, and D.N. Granger. 1991. Nitric oxide: an endogenous modulator of leukocyte adhesion. Proc. Natl. Acad. Sci. USA. 88:46514655 .

2. May, G.R., P. Crook, P.K. Moore, and C.P. Page. 1991. The role of nitric oxide as an endogenous regulator of platelet and neutrophil activation within the pulmonary circulation of the rabbit. Br. J. Pharmacol. 102:759-763.

3. Ma, X., A.S. Weyrich, D.J. Lefer, and A.M. Lefer. 1993. Diminished basal nitric oxide release after myocardial ischemia and reperfusion promotes neutrophil adherence to coronary endothelium. Circ. Res. 72:403-412.

4. Nishida, J., R.S. McCuskey, D. McDonnell, and E.S. Fox. 1994. Protective role of nitric oxide in hepatic microcirculatory dysfunction during endotoxemia. Am. J. Physiol. 267:G1135-G1141.

5. Niu, X.-F., C.W. Smith, and P. Kubes. 1994. Intracellular oxidative stress induced by nitric oxide synthesis inhibition increases endothelial cell adhesion to neutrophils. Circ. Res. 74:1133-1140.

6. Akimitsu, T., and R.J. Korthuis. 1994. Leukocyte adherence (LA) induced by inhibition of nitric oxide (NO) production in skeletal muscle. FASEB (Fed. Am. Soc. Exp. Biol.) J. 8:A1032.

7. Gauthier, T.W., K.L. Davenpeck, and A.M. Lefer. 1994. Nitric oxide attenuates leukocyte-endothelial interaction via P-selectin in splanchnic ischemia-reperfusion. Am. J. Physiol. 267:G562-G568.

8. De Caterina, R., P. Libby, H.B. Peng, V.J. Thannickal, T.B. Rajavashisth, M.A. Gimbrone, Jr., W.S. Shin, and J.K. Liao. 1995. Nitric oxide decreases cytokine-induced endothelial activation. Nitric oxide selectively reduces endothelial expression of adhesion molecules and proinflammatory cytokines. J. Clin. Invest. 96:60-68.

9. Tsao, P.S., R. Buitrago, J.R. Chan, and J.P. Cooke. 1996. Fluid flow inhibits endothelial adhesiveness. Nitric oxide and transcriptional regulation of VCAM-1. Circulation. 94:1682-1689.

10. Kurose, I., R. Wolf, M.B. Grisham, and D.N. Granger. 1994. Modulation of ischemia/reperfusion-induced microvascular dysfunction by nitric oxide. Circ. Res. 74:376-382.

11. Lefer, A.M., P.S. Tsao, D.J. Lefer, and X. Ma. 1991. Role of endothelial dysfunction in the pathogenesis of reperfusion injury after myocardial ischemia. FASEB (Fed. Am. Soc. Exp. Biol.) J. 5:2029-2034.

12. Liu, S.F., I.M. Adcock, R.W. Old, P.J. Barnes, and T.W. Evans. 1996 Differential regulation of the constitutive and inducible nitric oxide synthase mRNA by lipopolysaccharide treatment in vivo in the rat. Crit. Care Med. 24: 1219-1225.

13. Lu, J., L.M. Schmiege, L. Kuo, and J.C. Liao. 1996. Downregulation of endothelial constitutive nitric oxide synthase expression by lipopolysaccharide. Biochem. Biophys. Res. Commun. 225:1-5.

14. Kavanagh, B.P., and R.G. Pearl. 1995. Inhaled nitric oxide in anesthesia and critical care medicine. Int. Anesthesiol. Clin. 33:181-210.

15. Roberts, J.D., J.R. Fineman, F.C. Morin III, P.W. Shaul, S. Rimar, M.D Schreiber, R.A. Polin, M.S. Zwass, M.M. Zayek, I. Gross, M.A. Heymann, and W.M. Zapol. 1997. Inhaled nitric oxide and persistent pulmonary hypertension of the newborn. The inhaled nitric oxide study group. N. Engl. J. Med. 336:605610

16. Keaney, J.F., Jr., D.I. Simon, J.S. Stamler, O. Jaraki, J. Scharfstein, J.A. Vita, and J. Loscalzo. 1993. NO forms an adduct with serum albumin that has endothelium-derived relaxing factor-like properties. J. Clin. Invest. 91:15821589.

17. Stamler, J.S., D.I. Simon, J.A. Osborne, M.E. Mullins, O. Jaraki, D.J. Singel, and J. Loscalzo. 1992. S-nitrosylation of proteins by nitric oxide: synthesis and characterization of novel biologically active compounds. Proc. Natl. Acad. Sci. USA. 89:444-448.

18. Jia, L., C. Bonaventura, J. Bonaventura, and J.S. Stamler. 1996. S-nitrosohaemoglobin: a dynamic activity of blood involved in vascular control. $\mathrm{Na}$ ture. 380:221-226.

19. Kubes, P., M. Jutila, and D. Payne. 1995. Therapeutic potential of inhib- 
iting leukocyte rolling in ischemia/reperfusion. J. Clin. Invest. 95:2510-2519.

20. House, S.D., and H.H. Lipowsky. 1987. Leukocyte-endothelium adhesion: microhemodynamics in mesentery of the cat. Microvasc. Res. 34:363-379.

21. Kurose, I., R. Wolf, M.B. Grisham, T.Y. Aw, R.D. Specian, and D.N. Granger. 1995. Microvascular responses to inhibition of nitric oxide production: role of active oxidants. Circ. Res. 76:30-39.

22. Gaboury, J.P., X.-F. Niu, and P. Kubes. 1996. Nitric oxide inhibits numerous features of mast cell-induced inflammation. Circulation. 93:318-326.

23. Kubes, P., E. Sihota, and M.J. Hickey. 1997. Endogenous but not exogenous nitric oxide decreases TNF- $\alpha$-induced leukocyte rolling. Am. J. Physiol. 273:G628-G635.

24. Ostrovsky, L., A.J. King, S. Bond, D. Mitchell, X.-F. Niu, and P. Kubes. 1998. A juxtacrine mechanism for neutrophil adhesion on platelets involves platelet-activating factor and a selectin-dependent activation process. Blood. 91:3028-3036.

25. Buttrum, S.M., R. Hatton, and G.B. Nash. 1993. Selectin-mediated rolling of neutrophils on immobilized platelets. Blood. 82:1165-1174.

26. Weber, C. and T.A. Springer. 1997. Neutrophil accumulation on activated, surface-adherent platelets in flow is mediated by interaction of Mac-1 with fibrinogen bound to alphaIIbbeta 3 and stimulated by platelet-activating factor. J. Clin. Invest. 100:2085-2093.

27. Kubes, P., G. Ibbotson, J.M. Russell, J.L. Wallace, and D.N. Granger. 1990. Role of platelet-activating factor in ischemia/reperfusion-induced leukocyte adherence. Am. J. Physiol. 259:G300-G305.

28. Lawrence, M.B., C.W. Smith, S.G. Eskin, and L.V. McIntire. 1990. Effect of venous shear stress on CD18-mediated neutrophil adhesion to cultured endothelium. Blood. 75:227-237.

29. Kanwar, S., B. Johnston, and P. Kubes. 1995. Leukotriene C4/D4 induces P-selectin and sialyl lewisx-dependent alterations in leukocyte kinetics in vivo. Circ. Res. 77:879-887.

30. Edwards, A.D. 1995. The pharmacology of inhaled nitric oxide. Arch Dis. Child. Fetal Neonatal. Ed. 72:F127-F130.

31. Rosenberg, A.A., J.P. Kinsella, and S.H. Abman. 1995. Cerebral hemodynamics and distribution of left ventricular output during inhalation of nitric oxide. Crit. Care Med. 23:1391-1397.

32. Adrie, C., K.D. Bloch, P.R. Moreno, W.E. Hurford, J.L. Guerrero, R. Holt, W.M. Zapol, H.K. Gold, and M.J. Semigran. 1996. Inhaled nitric oxide increases coronary artery patency after thrombolysis. Circulation. 94:1919-1926.

33. Payne, D., and P. Kubes. 1993. Nitric oxide donors reduce the rise in reperfusion-induced intestinal mucosal permeability. Am. J. Physiol. 265:G189G195.

34. Kubes, P., P.H. Reinhardt, D. Payne, and R.C. Woodman. 1995. Excess nitric oxide does not cause cellular, vascular or mucosal dysfunction in the cat small intestine. Am. J. Physiol. 269:G34-G41.

35. Bloomfield, G.L., L.B. Sweeney, B.J. Fisher, C.R. Blocher, M.M. Sholley, J.H. Sugerman, and A.A. Fowler III. 1997. Delayed administration of inhaled nitric oxide preserves alveloar-capillary membrane integrity in porcine gram-negative sepsis. Arch. Surg. 132:65-75.

36. Gessler, P., T. Nebe, A. Birle, W. Mueller, and W. Kachel. 1996. A new side effect of inhaled nitric oxide in neonates and infants with pulmonary hypertension: functional impairment of the neutrophil respiratory burst. Intensive Care Med. 22:252-258.

37. Takahashi, Y., H. Kobayashi, N. Tanaka, T. Sato, N. Takizawa, and T. Tomita. 1998. Nitrosylhemoglobin in blood of normoxic and hypoxic sheep during nitric oxide inhalation. Am. J. Physiol. 274:H349-H357.

38. Lee J.S., C. Adrie, H.J. Jacob, J.D. Roberts, W.M. Zapol, and K.D. Bloch. 1996. Chronic inhalation of nitric oxide inhibits neointimal formation after balloon-induced arterial injury. Circ. Res. 78:337-342. 